\title{
Evolution of Reproductive Disorders Related to Celiac Disease under Gluten-free Diet
}

\author{
Aomari Ayoub*, Mohammed Firwana, Amina Amjahdi, Anass Rahaoui, Imane Benelbarhdadi, Ajana Fatima Zahra \\ Department of Gastroenterology and Liver Diseases, Medicine C, IBN SINA University Hospital Rabat Morocco \\ *Corresponding author: Ayoub.medinterne@gmail.com
}

\begin{abstract}
Introduction: Celiac disease (CD) is an autoimmune enteropathy induced by the ingestion of gluten (wheat, barley, rye). The classical form has become a minority. Currently, the most frequent forms of presentation are extradigestive with various manifestations, among others, reproductive disorders. The aim of our study is to assess the frequency of these disorders in $\mathrm{CD}$ and their evolution under gluten-free diet (GFD). Materials and methods: Descriptive retrospective study of 173 patients with CD followed in the department of diseases of the digestive tract "Médecine C" of the Ibn Sina Hospital in Rabat, over a period of 18 years. Result: In 173 patients with CD, 58 patients $(28,9 \%)$ had reproductive disorders. There are 53 women and 5 men. The average age was 32,25 years. The diagnosis of CD is based on histology and serology. The Reproductive problems were never isolated but always associated with other digestive or extra-digestive signs at the time of diagnosis of CD. These disorders are represented by: delayed puberty in 11 patients (19\%), secondary amenorrhea in 13 patients $(22.4 \%)$, irregular menstrual in 12 patients $(20.6 \%)$, absence of development of secondary sex characteristics in 8 patients $(12.5 \%)$, spontaneous abortions in 7 patients $(10.9 \%)$, menometrorrhagia in 4 patients $(13.8 \%)$, primary sterility in 5 patients $(8.6 \%)$, early menopause in 6 patients $(10.3 \%)$, premature delivery in 3 patients $(5 \%)$, primary amenorrhea in 2 patients (3.4\%) and intrauterine fetal death in one case (1.7\%). All our patients have had a GFD. 15 patients lost to follow-up, two patients died and 12 patients undergoing follow-up. The remaining 29 patients, the evolution of reproductive disorders under GFD was favorable in 26 patients $(90 \%)$, with the normalization of cycles in 15 patients, resumption of cycles in 6 patients, development of secondary sex characteristics in 2 patients, fertility resumption in one case, initiation of cycles after primary amenorrhea in one case and delivery of a newborn at term after premature deliveries in one case. The evolution was unfavorable in 3 patients with the notion of miscarriage 4 years after the start of the GFD in one patient and the absence of cycle resumption in two patients. Conclusion: The reproductive disorders associated with $\mathrm{CD}$ are frequent and varied. In our study, these disorders responded very well under a GFD, conducted in $90 \%$ of patients. These disorders are thus reversible under this diet.
\end{abstract}

Keywords: celiac disease, reproductive disorders, gluten- free diet

Cite This Article: Aomari Ayoub, Mohammed Firwana,Amina Amjahdi, Anass Rahaoui, Imane Benelbarhdadi, and Ajana Fatima Zahra, "Evolution of Reproductive Disorders Related to Celiac Disease under Gluten-free Diet." International Journal of Celiac Disease, vol. 5, no. 2 (2017): 69-71. doi: 10.12691/ijcd-5-2-8.

\section{Introduction}

Celiac disease (CD) is an autoimmune enteropathy induced by the ingestion of gluten (wheat, barley, rye) in genetically predisposed subjects leading to atrophic lesions of the small bowel, regressive under gluten-free diet (GFD). The classical form has become a minority. Currently, the most frequent forms of presentation are extradigestive with various manifestations, among others, reproductive disorders [1]. The aim of our study is to assess the frequency of these disorders in CD and their evolution under a GFD.

\section{Materials and Methods}

Descriptive retrospective study of 173 patients with CD followed in the department of diseases of the digestive tract "Médecine C" of the Ibn Sina Hospital in Rabat, over a period of 18 years.

\section{Result}

In 173 patients with $\mathrm{CD}, 58$ patients $(28,9 \%)$ had reproductive disorders. There are 53 women and 5 men. The average age was 32,25 years. The diagnosis of CD is based on histology (Severe or partial villous atrophy with intraepithelial lymphocytosis exceeding 30\%) and serology (Antigliadin and/or antiendomysium and/or antitransglutaminase positive antibodies). The Reproductive problems were never isolated but always associated with other digestive or extra-digestive signs at the time of diagnosis of CD. These disorders are represented by: delayed puberty in 11 patients (19\%), secondary amenorrhea in 13 patients $(22.4 \%)$, irregular menstrual in 12 patients $(20.6 \%)$, absence of development 
of secondary sex characteristics in 8 patients $(12.5 \%)$, spontaneous abortions in 7 patients (10.9\%), menometrorrhagia in 4 patients $(13.8 \%)$, primary sterility in 5 patients $(8.6 \%)$, early menopause in 6 patients $(10.3 \%)$, premature delivery in 3 patients $(5 \%)$, primary amenorrhea in 2 patients $(3.4 \%)$ and intrauterine fetal death in one case $(1.7 \%)$

All our patients have had a GFD. 15 patients lost to follow-up, two patients died and 12 patients undergoing follow-up. The remaining 29 patients, the evolution of reproductive disorders under GFD was favorable in 26 patients $(90 \%)$, with the normalization of cycles in 15 patients, resumption of cycles in 6 patients, development of secondary sex characteristics in 2 patients, fertility resumption in one case, initiation of cycles after primary amenorrhea in one case and delivery of a newborn at term after premature deliveries in one case. The evolution was unfavorable in 3 patients with the notion of miscarriage 4 years after the start of the GFD in one patient and the absence of a cycle resumption in two patients.

\section{Discussion}

$\mathrm{CD}$ is a mucosal disorder of the small intestine that may be triggered by dietary exposure to gluten in genetically-susceptible individuals. The disorder is often associated with diarrhea, malabsorption and weight loss along with other extra-intestinal complications. Reproductive changes have been described, including impaired fertility and adverse pregnancy outcomes possibly related to immune-mediated mechanisms or nutrient deficiency. Other possible pathogenetic factors that may alter placental function include maternal $\mathrm{CD}$ autoantibodies binding to placental transglutaminase, and genetic mutations that may facilitate microthrombus formation. Reports noting activation during pregnancy or the puerperium may be important, and suggest that CD may also be hypothetically precipitated by maternal exposure to one or more fetal antigens. In recent years, there has been an increased recognition of possible changes in male and female fertility in $\mathrm{CD}$ as well as the potential for adverse outcomes in pregnancy and the post-partum period that may lead to miscarriages and premature low birth weight fetal deliveries (Table 1) [2].

Delayed onset of menarche, amenorrhea, early menopause, recurrent abortions and reduced rates of pregnancy in CD may reflect an impairment of fertility. In 74 celiac patients from the United Kingdom [3], the reproductive period was longer for those on a GFD compared to those not on a diet but maternal health was not seriously impaired. A lower incidence of spontaneous abortions in celiacs on a GFD was also recorded. Similar results were reported in an Italian studyn [4]. In consecutively diagnosed celiacs compared to age- and "sexual behavior"-matched healthy controls, there was a significant delay in the mean age of menarche in untreated celiac patients (13.5 years compared to 12.1 years). Amenorrhea and repeated abortions were more common in the celiac group, but onset of menopause did not significantly differ. Studies from Poland and Italy $[5,6]$ also evaluated menarcheal age of celiac girls with reference to maternal menarcheal age. In one [5], menarcheal age of celiac girls appeared to be regulated by a GFD, while in the other [6], menarcheal age in CD was not delayed, but was affected by maternal menarcheal age. A further evaluation from the United Kingdom [7], suggested that celiacs are subfertile with an increased incidence of stillbirths and perinatal deaths. However, after diagnosis of CD and treatment with a GFD, some markers of infertility (e.g. miscarriage rates) may be corrected. Studies estimating prevalence of male infertility in $\mathrm{CD}$ have been rare. However, two more recent Italian publications have explored sexual behavior [8,9]. In one study, sexual behaviors in treated and untreated $\mathrm{CD}$ patients were examined using a questionnaire and compared to healthy controls [8]. Sexual satisfaction, including frequency of intercourse, was reduced in celiac patients, but improved after a year of treatment with a GFD. In the other [9], sexual habits appeared to be very different in celiacs who were never treated with a GFD. CD, especially if untreated, appears to increase the risk of repeated miscarriages and premature deliveries, and impaired fetal growth with reduced birthweight [10]. In addition, adverse effects on the mother may also occur, as indicated by a recent German study which demonstrated that the rate of cesarean delivery was increased if the parents had CD compared to other digestive disease controls as well as controls from eye or dental outpatient clinics [11]. In a case-control study from Italy that evaluated 94 untreated and 31 treated celiacs, the relative risks of either abortion or delivering a low birthweight baby were increased while the duration of breast feeding was significantly reduced [12]. All of these changes were apparently corrected with a GFD [12]. Higher incidences of either miscarriages or spontaneous abortions were also recorded from other centers located in different countries including Argentina, Italy and the United Kingdom $[3,4,7,13]$.

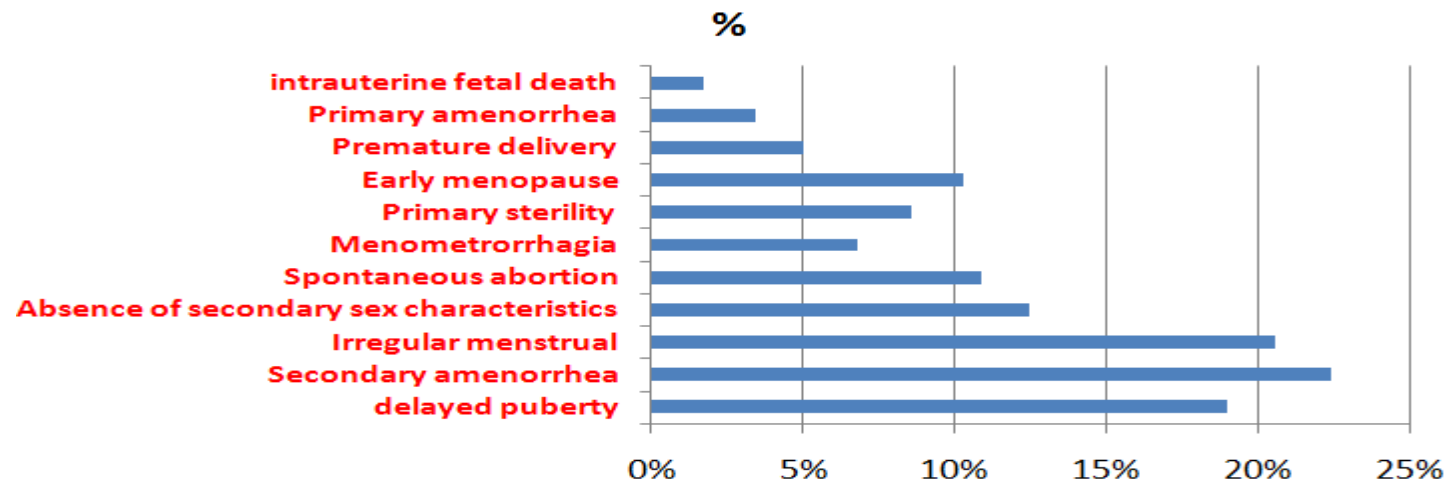

Figure 1. Percentage of reproductive disorders 
Table 1. Reproductive changes in celiac disease

\begin{tabular}{ll}
\hline Altered female fertility & Delayed onset of menarche, amenorrhea, early menopause, recurrent abortions, reduced rates of pregnancy \\
\hline Altered male fertility & $\begin{array}{l}\text { Gonadal dysfunction, altered sperm morphology and motility, reduced sexual activity } \\
\text { Repeated miscarriages, premature delivery an impaired fetal growth with low birth weight, abnormal } \\
\text { placental function }\end{array}$ \\
Alterations in pregnancy & Hormonal or immune changes \\
\hline
\end{tabular}

\section{Conclusion}

The reproductive disorders associated with $\mathrm{CD}$ are frequent and varied. In our study, these disorders responded very well under a GFD, conducted in $90 \%$ of patients. These disorders are thus reversible under this diet.

\section{References}

[1] Samasca G, Sur G, Lupan I, Tilinca M, Deleanu D. Celiac disease as an autoimmune condition. Cent Eur J Immunol 2014; 29(3): 396-399.

[2] Freeman HJ. Reproductive changes associated with celiac desease. World J Gastroenterol 2010; 16(46) 5810-5814.

[3] Ferguson R, Holmes GK, Cooke WT. Coeliac disease, fertility, and pregnancy. Scand J Gastroenterol. 1982; 17: 65-68.

[4] Molteni N, Bardella MT, Bianchi PA. Obstetric and gynecological problems in women with untreated celiac sprue. J Clin Gastroenterol. 1990; 12: 37-39.

[5] Rujner J. Age at menarche in girls with celiac disease Ginekol Pol. 1999; 70: 359-362
[6] Sferlazzas C, Arrigo T, Salzano G, Pellegrino S. Menarcheal age in celiac disease may not be delayed and may be irrespective of age at diagnosis and dietary management. J Endocrinol Invest. 2008; 31: 432-435.

[7] Sher KS, Mayberry JF. Female fertility, obstetric and gynaecological history in coeliac disease. A case control study. Digestion. 1994; 55: 243-246.

[8] Ciacci C, De Rosa A, de Michele G, Savino G. Sexual behaviour in untreated and treated coeliac patients. Eur J Gastroenterol Hepatol. 1998; 10: 649-651.

[9] Ciacci C, Iovino P, Amoruso D, Siniscalchi M. Grown-up coeliac children: the effects of only a few years on a gluten-free diet in childhood. Aliment Pharmacol Ther. 2005; 21: 421-429.

[10] Ogborn AD. Pregnancy in patients with coeliac disease. Br J Obstet Gynaecol. 1975; 82: 293-296.

[11] Decker E, Engelmann G, Findeisen A, Gerner P. Cesarean delivery is associated with celiac disease but not inflammatory bowel disease in children. Pediatrics. 2010; 125: e1433-e1440.

[12] Ciacci C, Cirillo M, Auriemma G, Di Dato G. Celiac disease and pregnancy outcome. Am J Gastroenterol. 1996; 91: 718-722.

[13] Smecuol E, Mauriño E, Vazquez H, Pedreira S, Niveloni S. Gynaecological and obstetric disorders in coeliac disease: frequent clinical onset during pregnancy or the puerperium. Eur J Gastroenterol Hepatol. 1996; 8: 63-89. 